\title{
Trends in Glucose Lowering Drug Utilization, Glycemic Control, and Severe Hypoglycaemia in Adults with Diabetes in Hong Kong 2002-2016
}

\section{Supplemental Material}

\section{Table of Contents}

Figure S1. Flow diagram of people with diabetes included in the study 2

Table S1. Trends in age- and sex- standardized percentage of CKD by eGFR (\%) among adults with diabetes in Hong Kong 2002-2016.

Table S2. Trends in insulin analogue use among adults with diabetes in Hong Kong 2002-2016.....

Figure S2. Age-stratified trends in standardized proportion (\%) of glucose lowering drugs among adults with diabetes in Hong Kong 2002-2016.

Figure S3. Sex-stratified trends in standardized proportion (\%) of glucose lowering drugs among adults with diabetes in Hong Kong 2002-2016.....

Figure S4. Age- and sex-stratified trends in annual mean HbA1c levels among adults with diabetes in Hong Kong 2002-2016

Figure S5. Age-stratified trends in standardized proportion of HbA1c category among adults with diabetes in Hong Kong 2002-2016

Figure S6. Treatment regimen stratified trends in standardized proportion of HbA1c category among adults with diabetes in Hong Kong 2002-2016.

Table S3. Joinpoint analysis of trends in standardized rate of severe hypoglycaemia with hospitalization (per 100 person-years) 2002-2016

Figure S7. Sensitivity analyses of trends in glucose lowering drugs (GLDs) utilization and annual mean HbA1c among adults with diabetes after excluding patients on no medications and those with newly diagnosed diabetes in Hong Kong 2002-2016

Figure S8. Sensitivity analyses of trends of mean HbA1c in the first year of diagnosis among newly diagnosed patients in Hong Kong 2002-2016 


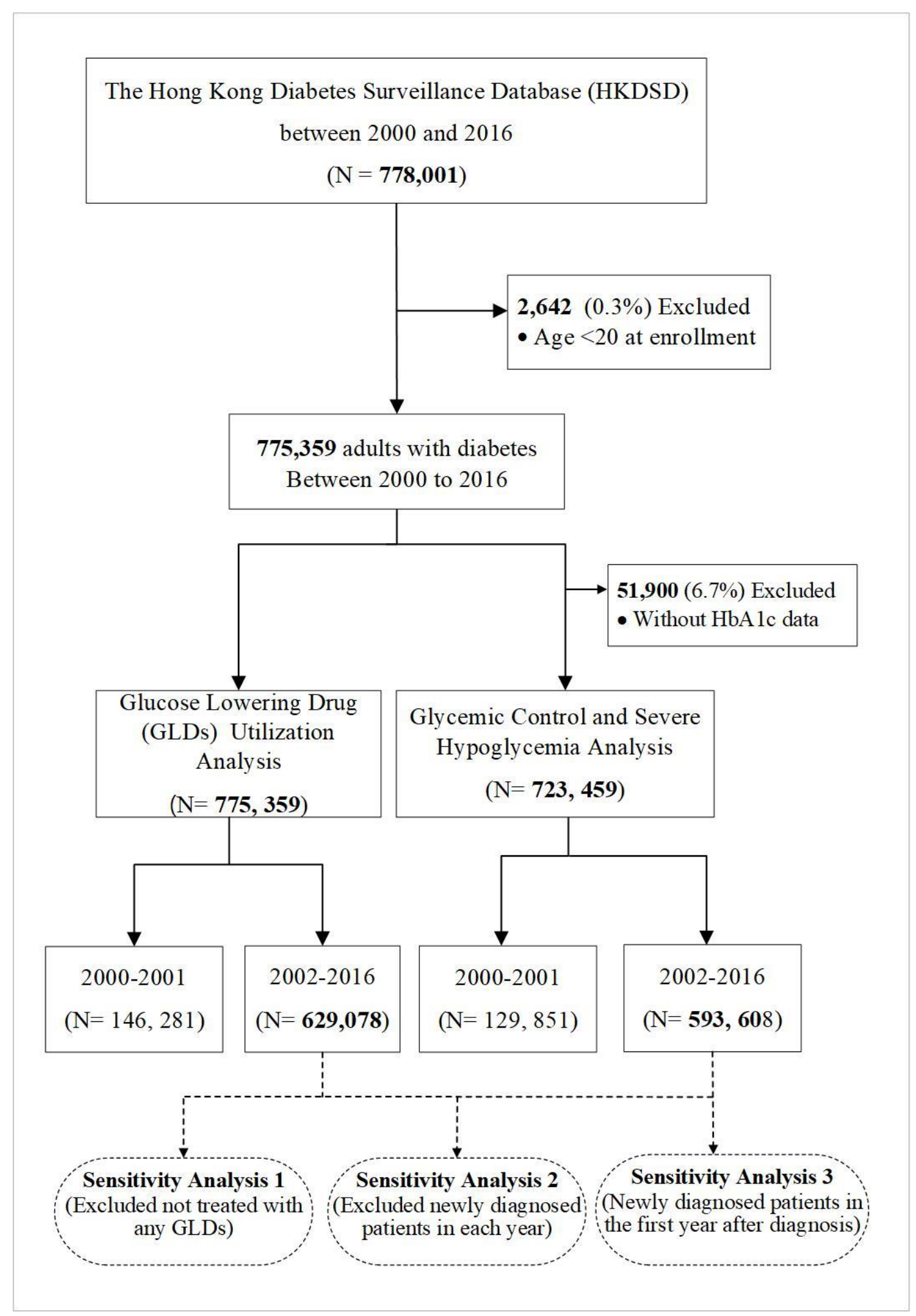

Figure S1. Flow diagram of people with diabetes included in the study 
Table S1. Trends in age- and sex- standardized percentage of CKD by eGFR (\%) among adults with diabetes in Hong Kong $2002-2016$

\begin{tabular}{|c|c|c|c|c|c|c|c|c|c|c|c|c|c|c|c|}
\hline & 2002 & 2003 & 2004 & 2005 & 2006 & 2007 & 2008 & 2009 & 2010 & 2011 & 2012 & 2013 & 2014 & 2015 & 2016 \\
\hline \multicolumn{16}{|c|}{ eGFR: $30-60 \mathrm{~mL} / \mathrm{min} / 1.73 \mathrm{~m}^{2}$} \\
\hline Overall & 30.1 & 29.1 & 27.4 & 26.5 & 25.1 & 23.2 & 22.4 & 22.6 & 20.9 & 19.7 & 19.4 & 19 & 18.9 & 18.5 & 18.7 \\
\hline Men & 27.6 & 27 & 26 & 25 & 24.1 & 22.4 & 21.4 & 21.7 & 20.2 & 19.2 & 19.1 & 18.8 & 18.7 & 18.4 & 18.5 \\
\hline Women & 32.3 & 31 & 28.8 & 27.9 & 26.1 & 24 & 23.2 & 23.4 & 21.6 & 20.1 & 19.6 & 19.1 & 19 & 18.6 & 18.8 \\
\hline $18-44$ & 3 & 3 & 3.2 & 3 & 2.9 & 2.9 & 2.8 & 3 & 2.8 & 2.6 & 2.5 & 2.4 & 2.4 & 2.4 & 2.3 \\
\hline $45-59$ & 8.7 & 8.1 & 7.8 & 7.2 & 6.8 & 6 & 5.7 & 5.7 & 4.9 & 4.5 & 4.5 & 4.5 & 4.6 & 4.6 & 4.9 \\
\hline $60-74$ & 30.7 & 29.6 & 27.5 & 26.1 & 24.2 & 21.7 & 20.6 & 20.5 & 18.3 & 17 & 16.7 & 16.2 & 15.9 & 15.5 & 15.6 \\
\hline $75+$ & 54.2 & 52.8 & 50.2 & 49.5 & 47.6 & 45.1 & 44.1 & 44.9 & 42.8 & 40.7 & 40.1 & 39.4 & 39.3 & 38.7 & 38.8 \\
\hline
\end{tabular}

eGFR: $<30 \mathrm{~mL} / \mathrm{min} / 1.73 \mathrm{~m}^{2}$

\begin{tabular}{|c|c|c|c|c|c|c|c|c|c|c|c|c|c|c|c|}
\hline Overall & 7 & 7.1 & 7 & 6.9 & 6.7 & 6.3 & 6.1 & 5.9 & 5.3 & 5 & 4.9 & 4.8 & 4.9 & 4.9 & 4.9 \\
\hline Men & 6 & 6.1 & 6.1 & 6.1 & 5.9 & 5.5 & 5.4 & 5.3 & 4.8 & 4.5 & 4.5 & 4.5 & 4.6 & 4.6 & 4.7 \\
\hline Women & 8 & 8.1 & 7.8 & 7.7 & 7.3 & 6.9 & 6.7 & 6.5 & 5.8 & 5.3 & 5.3 & 5.2 & 5.2 & 5.1 & 5.1 \\
\hline $18-44$ & 2 & 1.9 & 2.1 & 2.1 & 2.2 & 2 & 2 & 2 & 1.8 & 1.8 & 1.8 & 2 & 1.9 & 1.9 & 1.9 \\
\hline $45-59$ & 2.7 & 2.8 & 2.8 & 2.8 & 2.6 & 2.5 & 2.4 & 2.4 & 2.1 & 2 & 2 & 2 & 2 & 2.1 & 2.1 \\
\hline $60-74$ & 6.2 & 6.1 & 6 & 5.9 & 5.6 & 5.1 & 4.9 & 4.6 & 4 & 3.6 & 3.5 & 3.5 & 3.5 & 3.5 & 3.5 \\
\hline $75+$ & 13.2 & 13.4 & 13.1 & 13 & 12.7 & 12.2 & 11.9 & 11.7 & 10.7 & 10.1 & 10.1 & 9.9 & 10.1 & 9.9 & 9.9 \\
\hline
\end{tabular}

CKD, chronic kidney disease; eGFR, estimated glomerular filtration rate 
Table S2. Trends in insulin analogue use among adults with diabetes in Hong Kong 2002-2016

(A) Trends in insulin analogue use as a proportion (\%) of total insulin use

\begin{tabular}{|c|c|c|c|c|c|c|c|c|c|c|c|c|c|c|c|}
\hline & 2002 & 2003 & 2004 & 2005 & 2006 & 2007 & 2008 & 2009 & 2010 & 2011 & 2012 & 2013 & 2014 & 2015 & 2016 \\
\hline All & 1.7 & 1.9 & 2.5 & 2.8 & 3.0 & 3.5 & 4.1 & 4.8 & 5.7 & 7.3 & 9.6 & 11.3 & 12.5 & 13.9 & 16.2 \\
\hline Men & 1.8 & 2.0 & 2.5 & 2.8 & 2.9 & 3.3 & 3.9 & 4.6 & 5.5 & 7.5 & 9.8 & 11.5 & 12.7 & 14.1 & 16.3 \\
\hline Women & 1.6 & 1.9 & 2.5 & 2.9 & 3.2 & 3.8 & 4.3 & 5.0 & 5.9 & 7.2 & 9.4 & 11.1 & 12.3 & 13.6 & 16.0 \\
\hline $20-44$ & 7.9 & 9.5 & 12.6 & 13.9 & 13.9 & 14.7 & 16.1 & 18.5 & 20.2 & 24.5 & 27.2 & 29.3 & 31.3 & 33.0 & 36.2 \\
\hline $45-59$ & 1.6 & 1.8 & 2.3 & 3.1 & 3.6 & 4.3 & 5.5 & 6.1 & 7.1 & 9.2 & 12.3 & 14.4 & 15.7 & 17.1 & 19.1 \\
\hline $60-74$ & 0.3 & 0.4 & 0.8 & 1.0 & 1.4 & 2.0 & 2.6 & 3.3 & 4.3 & 5.9 & 8.5 & 10.0 & 11.5 & 12.9 & 15.2 \\
\hline $75+$ & 0.2 & 0.1 & 0.3 & 0.4 & 0.5 & 0.9 & 1.0 & 1.6 & 2.5 & 3.2 & 4.7 & 6.2 & 7.2 & 8.7 & 11.3 \\
\hline
\end{tabular}

(B) Trends in age- and sex-standardized proportion (\%) of insulin analogue use

\begin{tabular}{|c|c|c|c|c|c|c|c|c|c|c|c|c|c|c|c|}
\hline & 2002 & 2003 & 2004 & 2005 & 2006 & 2007 & 2008 & 2009 & 2010 & 2011 & 2012 & 2013 & 2014 & 2015 & 2016 \\
\hline Overall & 0.2 & 0.2 & 0.2 & 0.3 & 0.3 & 0.4 & 0.4 & 0.5 & 0.6 & 0.9 & 1.2 & 1.4 & 1.5 & 1.8 & 2.0 \\
\hline Men & 0.2 & 0.2 & 0.2 & 0.3 & 0.3 & 0.4 & 0.4 & 0.5 & 0.6 & 0.9 & 1.2 & 1.5 & 1.6 & 1.9 & 2.2 \\
\hline Women & 0.2 & 0.2 & 0.2 & 0.3 & 0.3 & 0.4 & 0.5 & 0.5 & 0.6 & 0.8 & 1.1 & 1.3 & 1.5 & 1.7 & 1.9 \\
\hline $20-44$ & 1.5 & 1.7 & 2.1 & 2.3 & 2.2 & 2.5 & 2.6 & 3.0 & 3.1 & 3.8 & 4.4 & 4.6 & 4.9 & 5.2 & 5.5 \\
\hline $45-59$ & 0.2 & 0.2 & 0.2 & 0.3 & 0.4 & 0.4 & 0.6 & 0.6 & 0.7 & 1.0 & 1.4 & 1.7 & 1.9 & 2.1 & 2.4 \\
\hline $60-74$ & 0.0 & 0.0 & 0.1 & 0.1 & 0.1 & 0.2 & 0.3 & 0.4 & 0.4 & 0.6 & 1.0 & 1.1 & 1.3 & 1.5 & 1.8 \\
\hline $75+$ & 0.0 & 0.0 & 0.0 & 0.0 & 0.1 & 0.1 & 0.1 & 0.2 & 0.3 & 0.4 & 0.5 & 0.7 & 0.8 & 1.0 & 1.4 \\
\hline
\end{tabular}



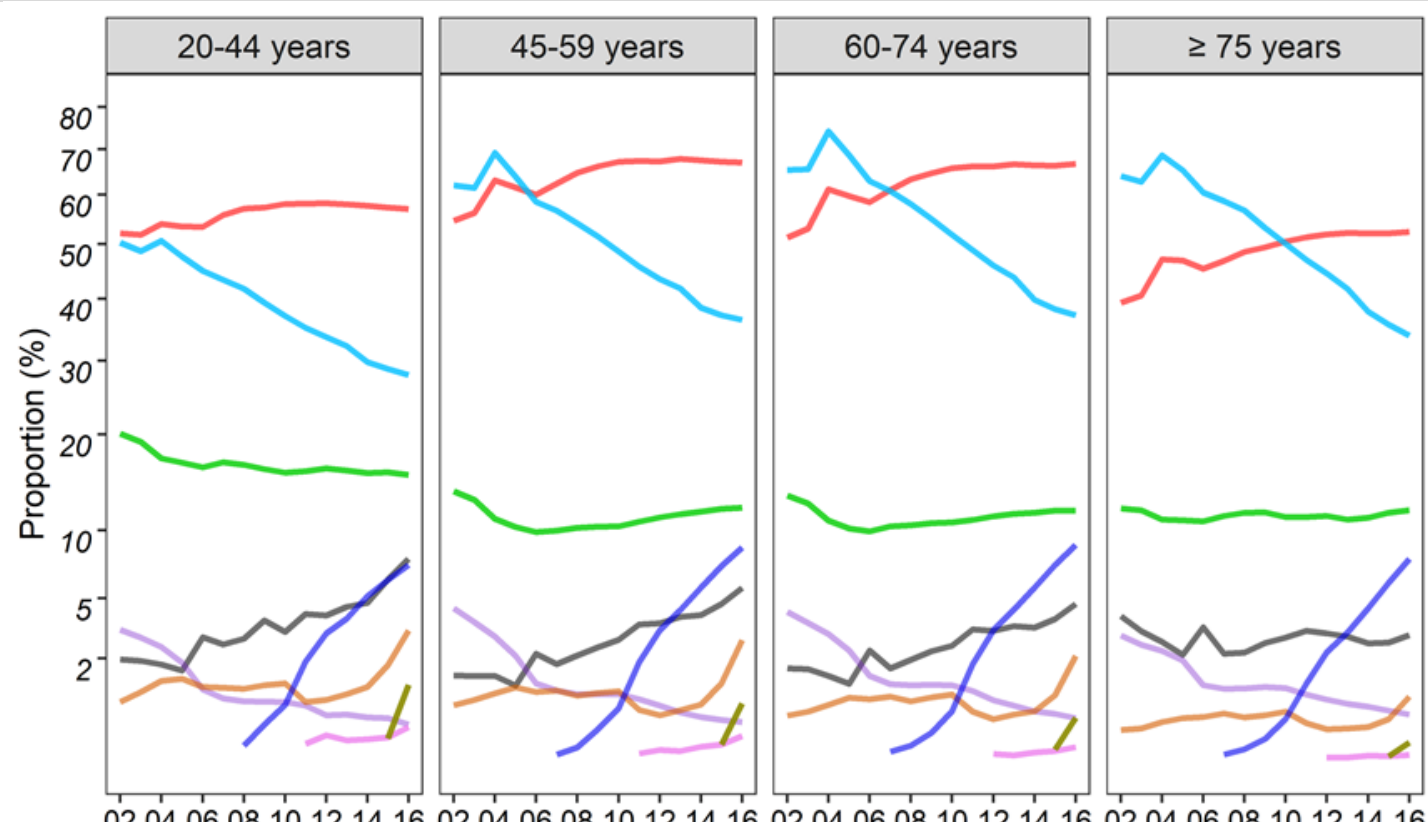

0204060810121416020406081012141602040608101214160204060810121416 Year

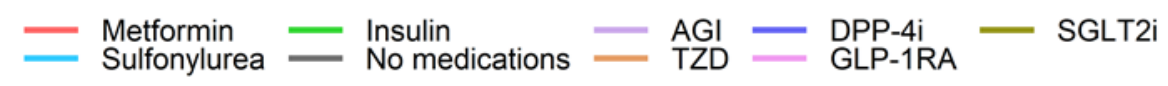

Figure S2. Age-stratified trends in standardized proportion (\%) of glucose lowering drugs among adults with diabetes in Hong Kong 2002-2016 AGIs, alpha glucosidase inhibitors; TZD, thiazolidinediones; DPP-4i, dipeptidyl peptidase-4 inhibitors; GLP-1RA, glucagon-like peptide-1 receptor agonists; SGLT2i, sodium-glucose co-transporter-2 inhibitors. 

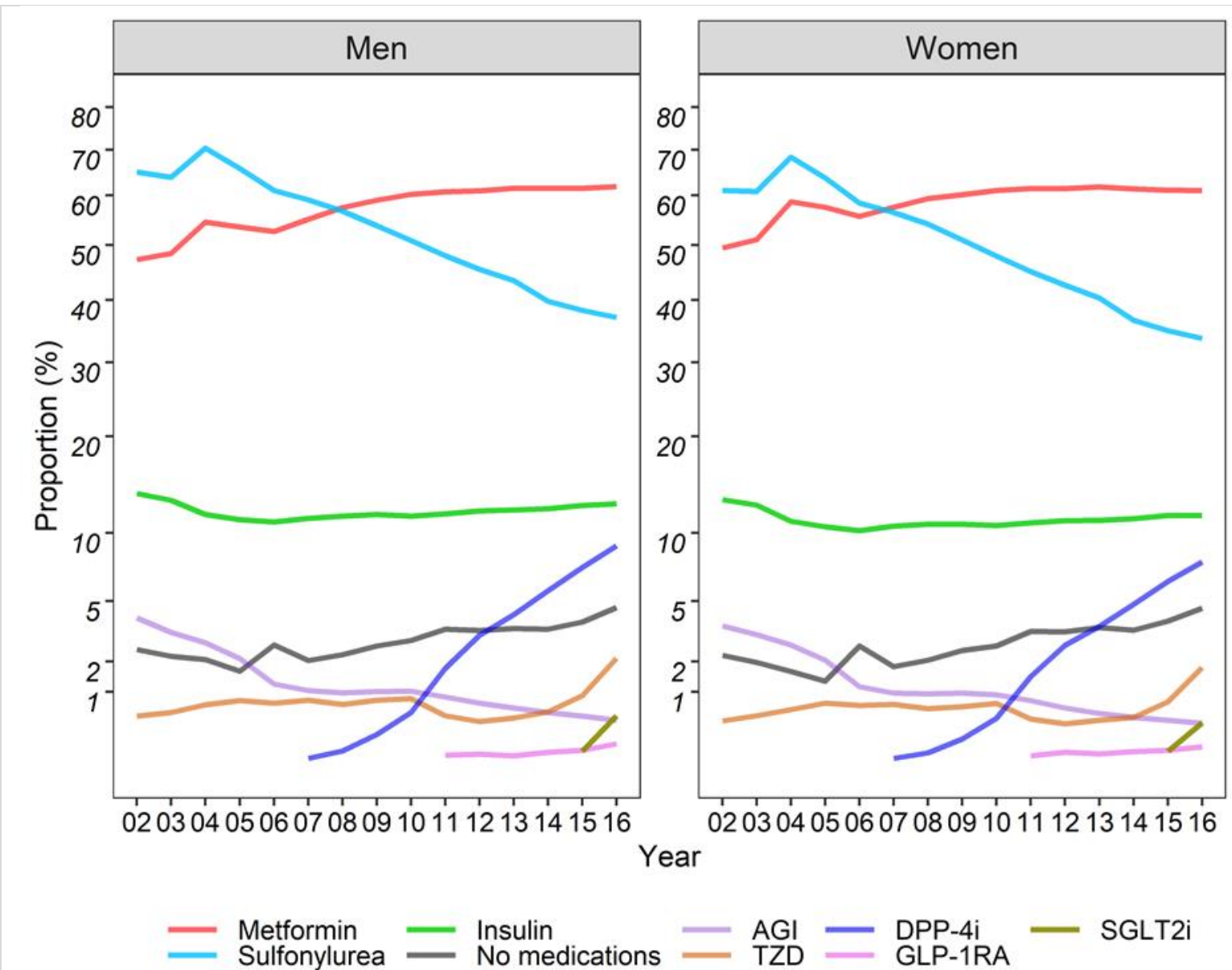

Figure S3. Sex-stratified trends in standardized proportion $(\%)$ of glucose lowering drugs among adults with diabetes in Hong Kong 2002-2016 AGIs, alpha glucosidase inhibitors; TZD, thiazolidinediones; DPP4i, dipeptidyl peptidase-4 inhibitors; GLP-1RA, glucagon-like peptide-1 receptor agonists; SGLT2i, sodiumglucose co-transporter-2 inhibitors. 


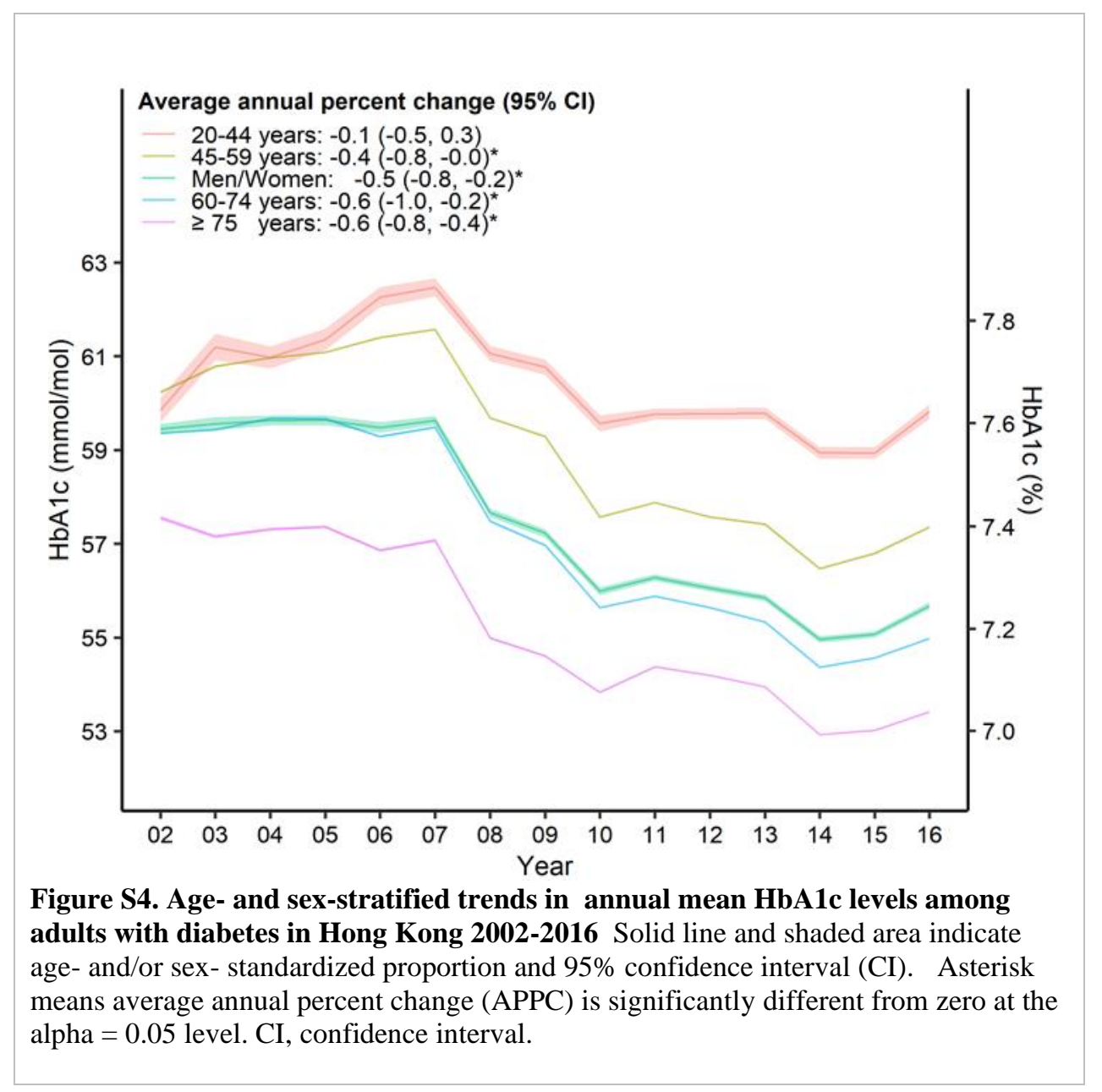




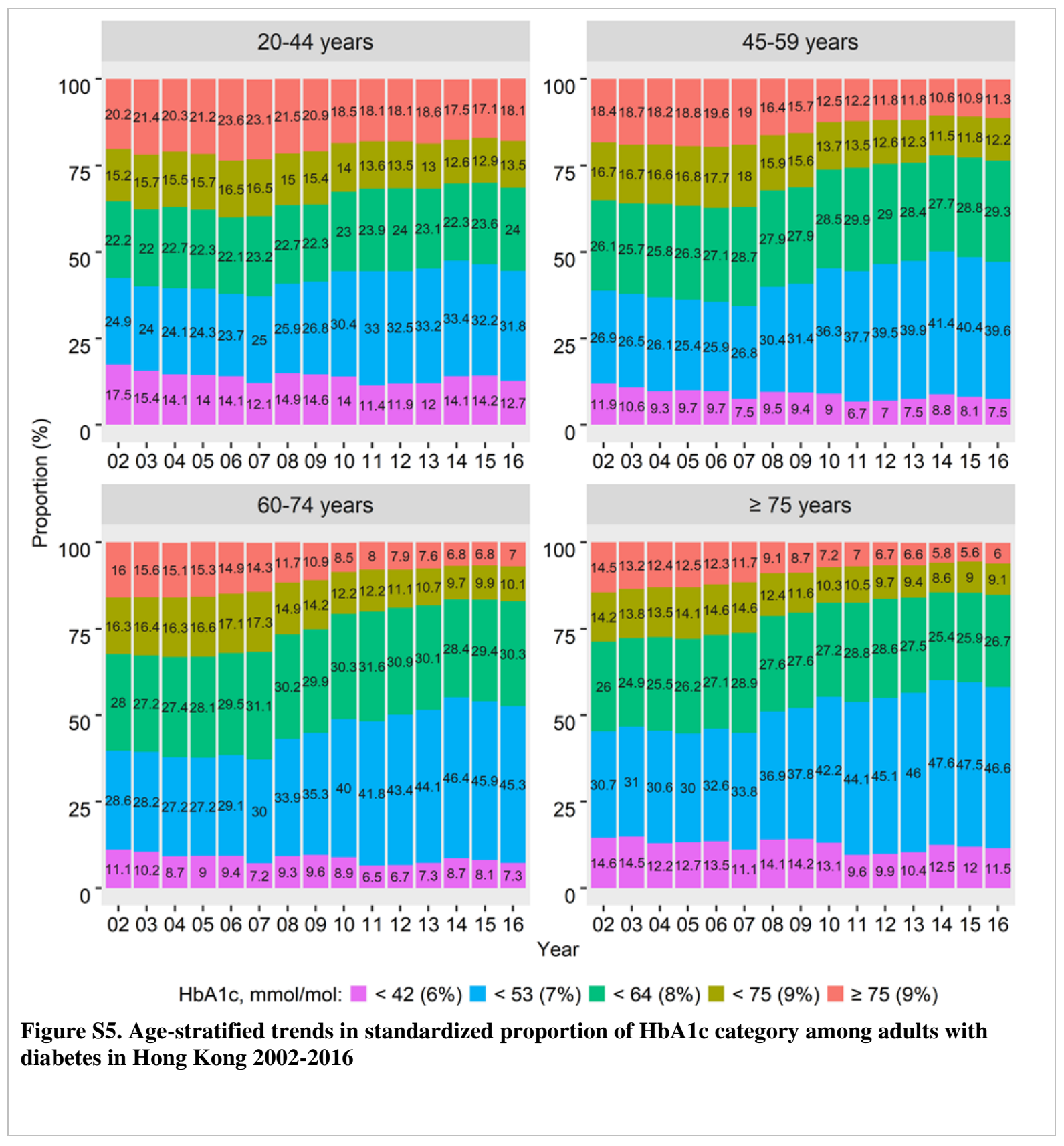




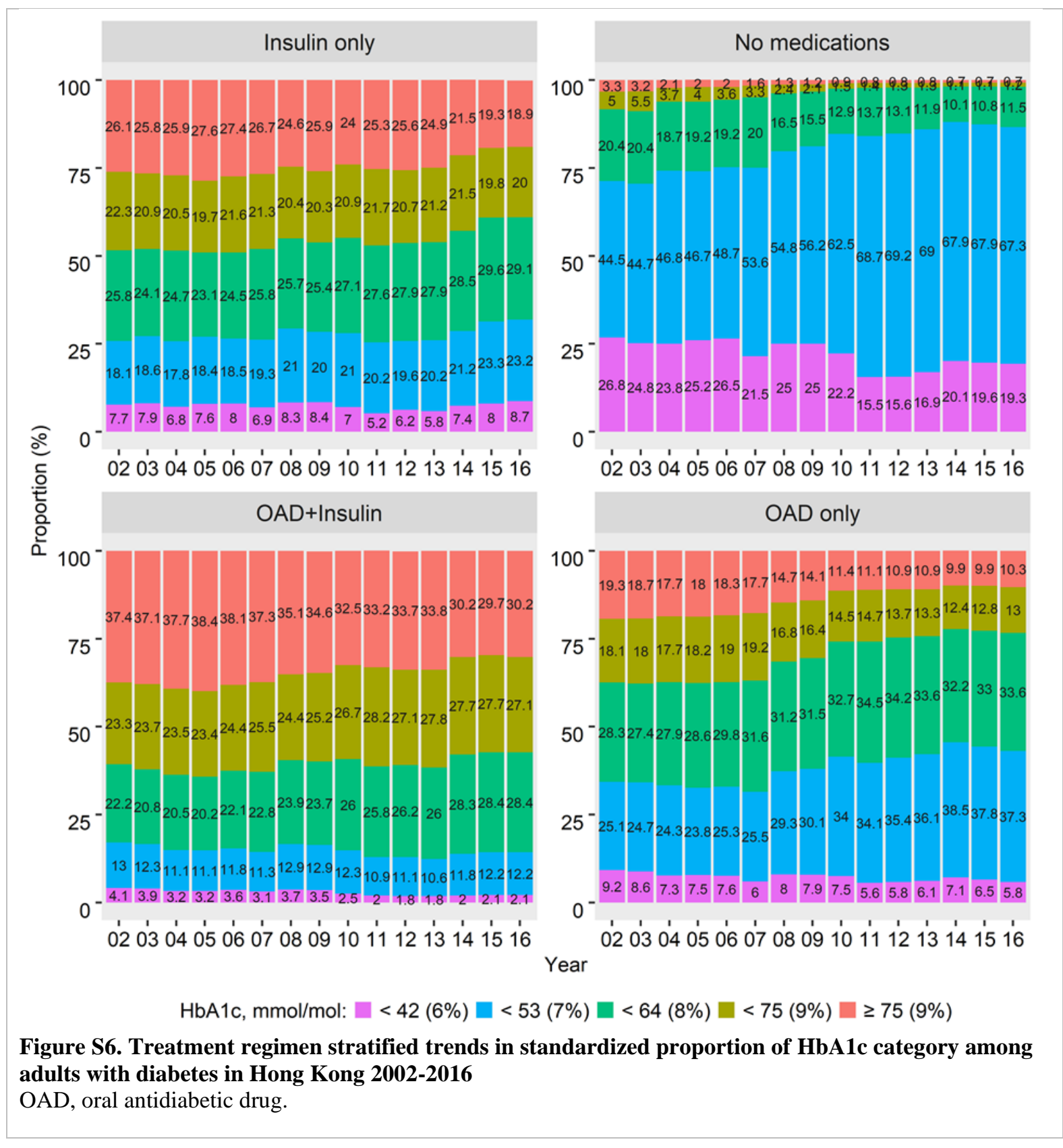


Table S3. Joinpoint analysis of trends in standardized rate of severe hypoglycaemia with hospitalization (per 100 person-years) $2002-2016$

\begin{tabular}{|c|c|c|c|c|c|c|c|c|c|}
\hline & \multicolumn{3}{|c|}{$\begin{array}{l}\text { Severe hypoglycaemia rate } \\
\text { (100 patient-years) }\end{array}$} & \multicolumn{2}{|l|}{ Period 1} & \multicolumn{2}{|l|}{ Period 2} & \multicolumn{2}{|l|}{ Period 3} \\
\hline & 2002 & 2006 & AAPC $(95 \% \mathrm{CI})$ & Year & $\mathrm{APC}(95 \% \mathrm{CI})$ & Year & $\operatorname{APC}(95 \% \mathrm{CI})$ & Year & $\mathrm{APC}(95 \% \mathrm{CI})$ \\
\hline Overall & 4.2 & 1.3 & $-8.3(-10.5,-6)^{*}$ & $2002-2006$ & $-22.5(-27.9,-16.7)^{*}$ & 2006-2010 & $9.4(2.8,16.5)^{*}$ & 2010-2016 & $-9.6(-11.7,-7.4)^{*}$ \\
\hline \multicolumn{10}{|l|}{ Sex } \\
\hline Men & 4.2 & 1.3 & $-8.1(-9.6,-6.5)^{*}$ & 2002-2006 & $-20.8(-24.7,-16.7)^{*}$ & 2006-2010 & $7.7(3.2,12.4)^{*}$ & 2010-2016 & $-9.3(-10.7,-7.8)^{*}$ \\
\hline Women & 4.3 & 1.3 & $-8.4(-11.6,-5)^{*}$ & 2002-2006 & $-23(-29.8,-15.6)^{*}$ & 2006-2009 & $15.7(1.9,31.3)^{*}$ & 2009-2016 & $-9.5(-12.3,-6.6)^{*}$ \\
\hline \multicolumn{10}{|c|}{ Age groups (years) } \\
\hline $20-44$ & 4.0 & 1.6 & $-6.5(-8.6,-4.3)^{*}$ & $2002-2005$ & $-25.1(-31.1,-18.6)^{*}$ & $2005-2010$ & $8.1(2.4,14.2)^{*}$ & 2010-2016 & $-6.7(-8.7,-4.6)^{*}$ \\
\hline $45-59$ & 3.0 & 0.8 & $-8.9(-11.5,-6.3)^{*}$ & $2002-2005$ & $-28.3(-34.1,-21.9)^{*}$ & $2005-2010$ & $8(0.2,16.3)^{*}$ & 2010-2016 & $-8.1(-10.5,-5.5)^{*}$ \\
\hline $60-74$ & 4.3 & 1.1 & $-9.7(-12.7,-6.6)^{*}$ & 2002-2006 & $-25(-31.4,-17.9)^{*}$ & 2006-2010 & $8.3(-1.1,18.7)$ & 2010-2016 & $-10.4(-13.4,-7.3)$ \\
\hline $75+$ & 5.9 & 2.2 & $-6.9(-9.5,-4.2)^{*}$ & 2002-2006 & $-16.5(-21.5,-11.2)^{*}$ & 2006-2009 & $15.2(3,28.8)^{*}$ & 2009-2016 & $-9.2(-11.6,-6.7)^{*}$ \\
\hline \multicolumn{10}{|c|}{ Treatment regimen } \\
\hline OAD only & 5.3 & 1.8 & $-7.8(-10.2,-5.3)^{*}$ & 2002-2006 & $-24.9(-30.6,-18.8)^{*}$ & 2006-2009 & $12.2(4.6,20.3)^{*}$ & $2009-2016$ & $-7.3(-9.7,-4.9)^{*}$ \\
\hline OAD + Insulin & 20.0 & 7.8 & $-6.6(-8.9,-4.3)^{*}$ & 2002-2006 & $-13.7(-20.1,-6.7)^{*}$ & 2006-2009 & $4(-2.5,10.9)$ & 2009-2016 & $-8.1(-10.2,-6)^{*}$ \\
\hline Insulin only & 12.1 & 8.4 & $-2.7(-4.7,-0.7)^{*}$ & 2002-2006 & $-8.2(-12.7,-3.5)^{*}$ & 2006-2010 & $11.5(4.5,18.9)^{*}$ & 2010-2016 & $-7.6(-9.5,-5.6)^{*}$ \\
\hline
\end{tabular}

* The Annual Percent Change (APC) and/or Average Annual Percent Change (APPC) is significantly different from zero at the alpha = 0.05 level. CI, confidence interval; $\mathrm{OAD}$, oral antidiabetic drug. 
A

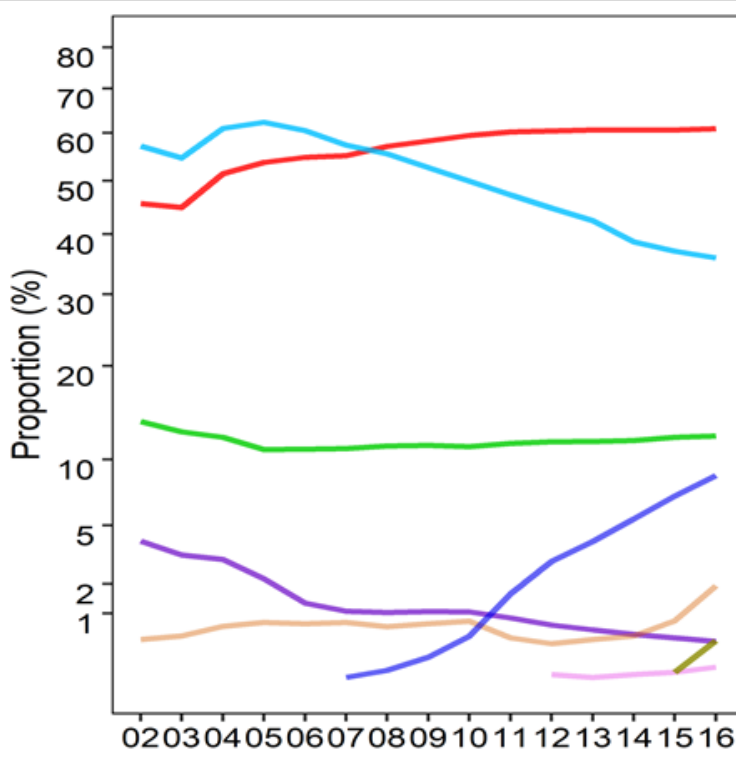

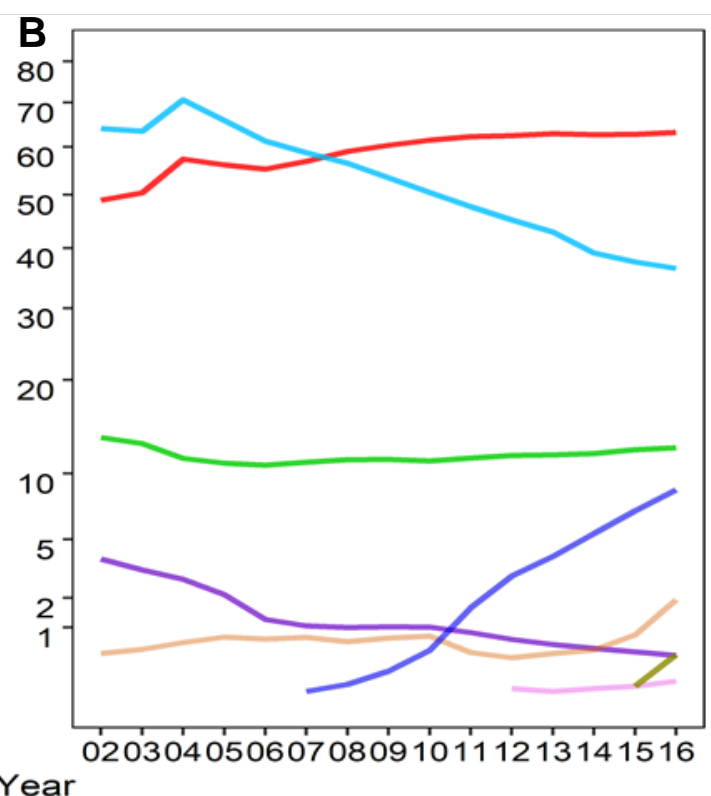

$\longrightarrow$ Metformin $\longrightarrow$ Sulfonylurea $\longrightarrow$ A $=$

\section{TZD - TLP-1RA}

C

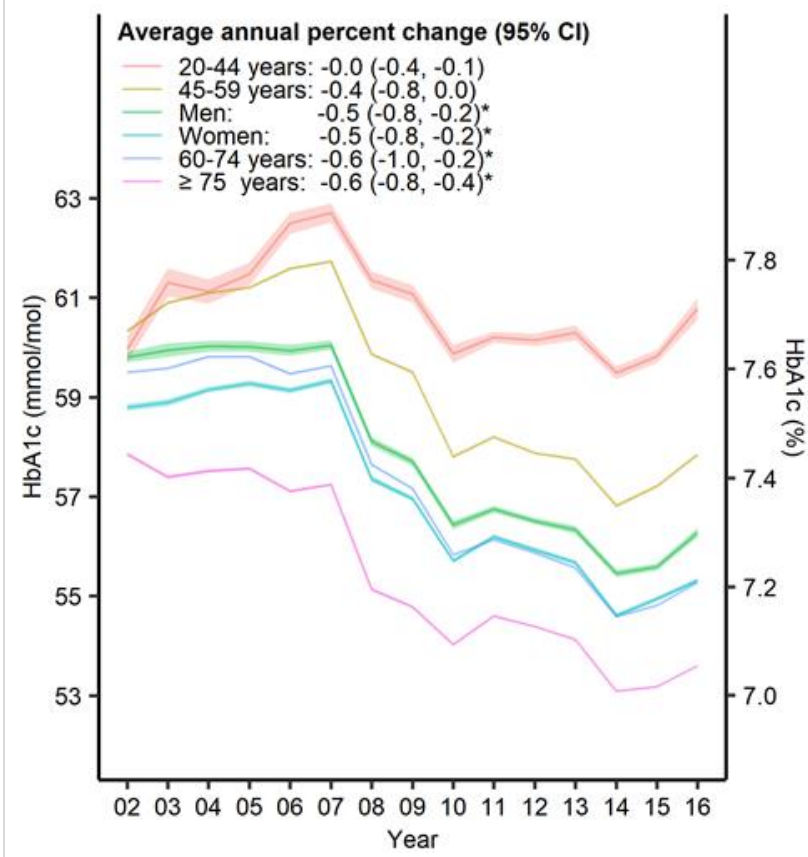

D

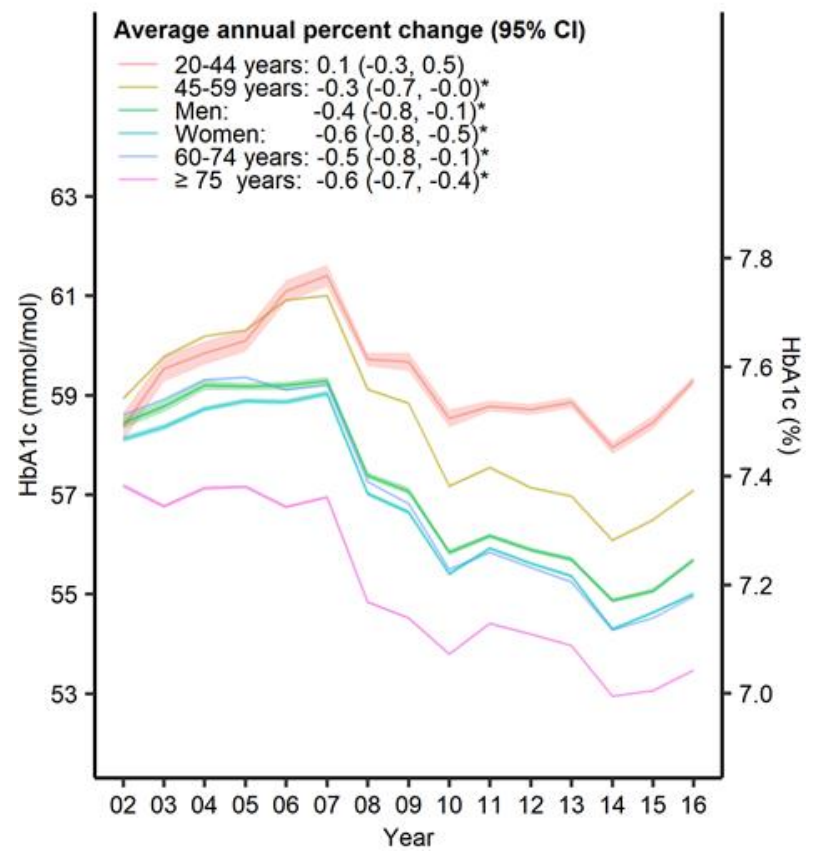

Figure S7. Sensitivity analyses of trends in glucose lowering drugs (GLDs) utilization and annual mean HbA1c among adults with diabetes after excluding patients on no medications and those with newly diagnosed diabetes in Hong Kong 2002-2016 (A) and (B): GLDs utilization pattern after excluding no medication patients, and newly diagnosed patients, respectively; (C) and (D): annual mean HbA1c after excluding no medication patients, and newly diagnosed patients, respectively. AGIs, alpha glucosidase inhibitors; TZD, thiazolidinediones; DPP-4i, dipeptidyl peptidase-4 inhibitors; GLP-1RA, glucagon-like peptide-1 receptor agonists; SGLT-2i, sodium-glucose co-transporter-2 inhibitors. Asterisk means average annual percent change (APPC) is significantly different from zero at the alpha $=0.05$ level. $\mathrm{CI}$, confidence interval. 

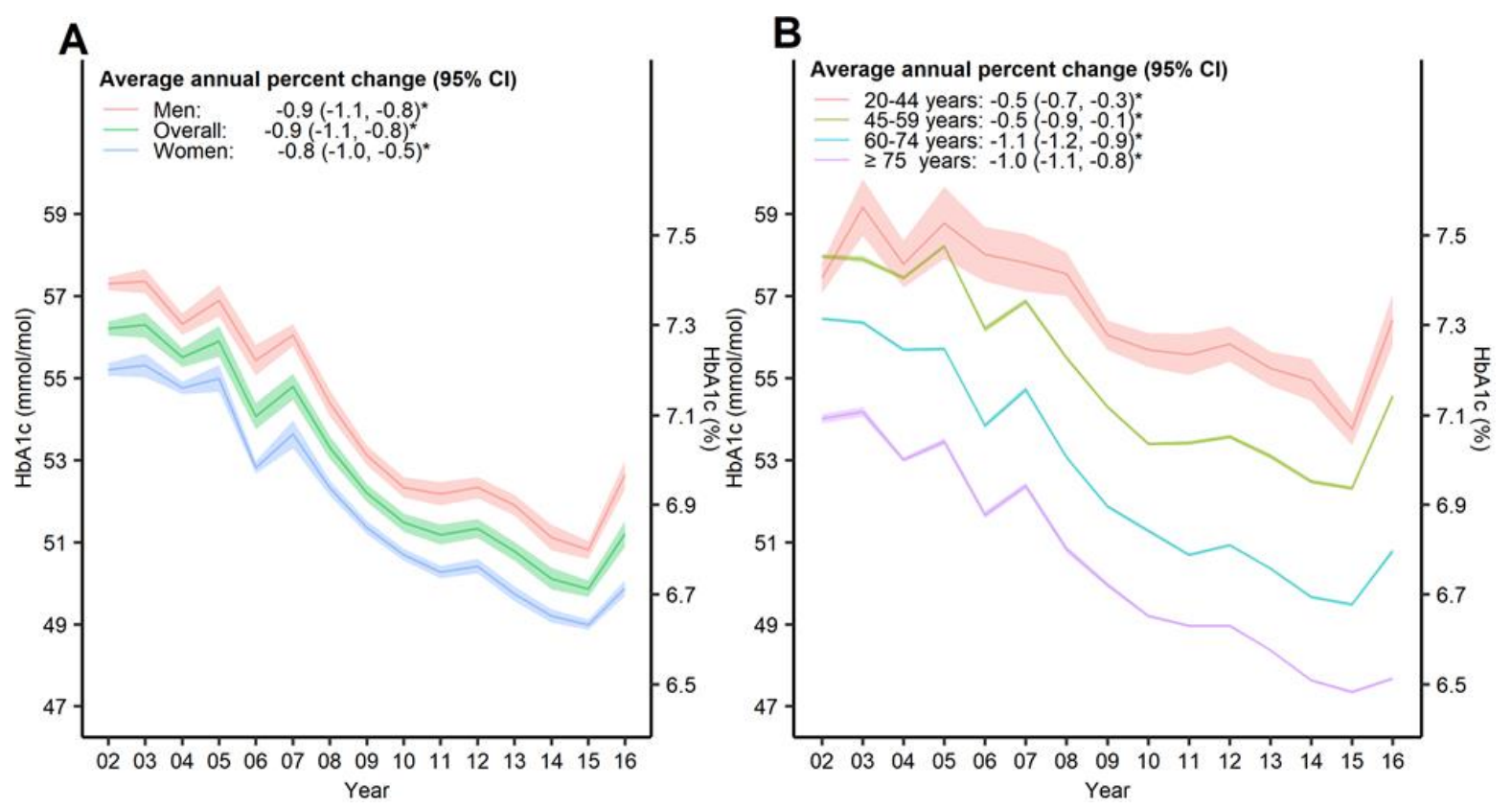

Figure S8. Sensitivity analyses of trends of mean HbA1c in the first year of diagnosis among newly diagnosed patients in Hong Kong 2002-2016 (A) overall and sex-stratified trends; (B) age-stratified trends. $*$ Average annual percent change (APPC) is significantly different from zero at the alpha $=0.05$ level. CI, confidence interval. 